\title{
Prognostic significance of urinary NGAL in chronic kidney disease
}

\author{
Munna Lal Patel' \\ Rekha Sachan ${ }^{2}$ \\ Ravi Misra ${ }^{3}$ \\ Ritul Kamal ${ }^{4}$ \\ Radhey Shyam ${ }^{5}$ \\ Pushpalata Sachan ${ }^{6}$ \\ 'Department of Medicine, King \\ George Medical University, Lucknow, \\ India; ${ }^{2}$ Department of Obstetrics \\ and Gynaecology, King George \\ Medical University, Lucknow, India; \\ ${ }^{3}$ Department of Internal Medicine, \\ King George Medical University, \\ Lucknow, India; ${ }^{4}$ Epidemiology \\ Division, Council of Scientific and \\ Industrial Research-Indian Institute \\ of Toxicology Research (CSIR-IITR), \\ Lucknow, India; ${ }^{5}$ Department of \\ Geriatric Intensive Care Unit, King \\ George Medical University, Lucknow, \\ India; 'Department of Physiology, \\ Career Institute of Medical Sciences, \\ Lucknow, India
}

This article was published in the following Dove Press journal: International Journal of Nephrology and Renovascular Disease 15 October 2015

Number of times this article has been viewed

Background: Chronic kidney disease (CKD) is a worldwide public health problem. Recently urinary NGAL (uNGAL) has been proven to be a useful (potentially ideal) biomarker for early detection of CKD. The aim of the present study was to examine the correlation of UNGAL with severity of renal impairment in CKD and to evaluate its prognostic value in these subjects.

Methods: This was a prospective study carried out over a period of 24 months in subjects with CKD due to primary chronic glomerulonephritis. New cases of CKD stage II, III, IV aged between 18 and 65 years were enrolled as per KDIGO (Kidney Disease: Improving Global Outcomes) guidelines 2012. A total of 90 subjects completed the study up to the end-point. The primary follow-up end-point was 18 months, or decreased glomerular filtration rate of less than $15 \mathrm{~mL} / \mathrm{min}$. Secondary follow-up end-point was the number of subjects who expired during this period.

Results: Multiple regression model of estimated glomerular filtration rate showed significant associations with log uNGAL $(\beta=0.38, P<0.001)$, Ca×PO4 $(\beta=0.60, P<0.001)$, hemoglobin $(\beta=0.37, P<0.001)$, urine protein $(\beta=0.34, P<0.001)$, serum albumin $(\beta=0.48, P<0.001)$, and systolic blood pressure $(\beta=0.76, P<0.001)$. Receiver operator curve for uNGAL considering the progression of CKD showed area under the curve for UNGAL was $0.878(95 \%$ confidence interval: $0.68-0.96)$. Cut-off value for uNGAL was $\log 3.5$ unit with a sensitivity of $93.08 \%$ and specificity of $71.43 \%$ for predicting the progression of CKD. Kaplan-Meier survival curve showed that patients with $\log$ uNGAL levels $<3.51$ unit had a survival rate of $71.4 \%$ while patients with NGAL level $>3.51$ unit had a renal survival rate of $14.7 \%$.

Conclusion: Our study result showed that UNGAL has a positive correlation with disease severity which signifies the prognostic importance of UNGAL in CKD.

Keywords: urinary NGAL, chronic kidney disease, prognostic significance

\section{Introduction}

Chronic kidney disease (CKD) is a worldwide public health problem, in view of both the number of patients and cost of treatment involved. Globally, CKD is the 12 th most common cause of death and the 17 th leading cause of disability. This is an underestimation as patients with CKD are more likely to die due to cardiovascular disease than to reach end-stage renal disease. Numerous risk factors for accelerated progression have been identified, ${ }^{1-4}$ with poorly controlled hypertension and heavy proteinuria being two of the best-recognized predictors. ${ }^{5}$

Diabetes and hypertension are the leading causes of CKD. ${ }^{6}$ Over the past 20 years Department of Medicine, King Georg Medical University, C-28, Sec-J Aliganj, Lucknow 226024, India Tel +9l 9839007000 Email patel.ml66@gmail.com it was thought that early diagnosis and treatment of kidney disease can prevent kidney disease progression. Thus, a biomarker of kidney damage which is able to indicate the presence of both early damage and identify patients at an increased risk

submit your manuscript | www.dovepress.com 
of progressive disease would affect kidney disease diagnosis and treatment.

Although serum creatinine is generally used as an index of renal function, creatinine is primarily a marker of glomerular filtration and this cannot be considered an ideal biomarker for the estimation of kidney injury, because it is insensitive and is influenced by muscle mass, sex, race, and medications. It is unreliable to diagnose renal tubular injury in the absence of significant reduction in the estimated glomerular filtration rate (eGFR). ${ }^{7,8}$ If by any investigative modalities disease is diagnosed in the early stage, disease progression can be prevented. NGAL has recently been proven to be a useful marker in CKD and it has the potential to be an ideal biomarker in early detection of CKD. The aim of the present study was to examine the correlation of urinary NGAL (uNGAL) with severity of renal impairment in CKD and to evaluate its prognostic value in these subjects.

\section{Materials and methods}

This was a prospective study carried out in the Department of Medicine in Nephrology Unit, King George Medical University, Lucknow, India over a period of 2 years from August 2012 to July 2014. After written informed consent and ethical clearance from King George's Medical University Lucknow, patients with CKD due to primary chronic glomerulonephritis with stable kidney function for at least for 5 months were enrolled in the study. All the new cases aged between 18 and 65 years with CKD stage II, III, IV were enrolled as per KDIGO (Kidney Disease: Improving Global Outcomes) guidelines 2012. ${ }^{9}$ A total of 102 subjects were enrolled in the study, 90 subjects completed the study up to end-point and 12 subjects dropped out from the study. After enrollment these patients were followed for 18 months. The diagnostic criteria of primary chronic glomerulonephritis were: the presence of glomerular proteinuria and/or hematuria lasting more than 1 year and exclusion of secondary or congenital glomerulonephritis. In our study disease progression was decided on the basis of declining eGFR or progression of CKD stage, ie, progression from stage II to stage III etc. The Modification of Diet in Renal Disease formula [GFR $\left(\mathrm{mL} / \mathrm{min} / 1.73 \mathrm{~m}^{2}\right)=175 \times\left(\mathrm{S}_{\mathrm{cr}}\right)^{-1.154} \times(\text { Age })^{-0.203} \times(0.742$ if female)] was used to calculate the eGFR. The staging criteria for CKD were defined as: stage II, renal damage with eGFR of $60-89 \mathrm{~mL} / \mathrm{min}$ per $1.73 \mathrm{~m}^{2}$; stage III, eGFR of $30-59 \mathrm{~mL} /$ min per $1.73 \mathrm{~m}^{2}$; and stage IV, eGFR of $15-29 \mathrm{~mL} / \mathrm{min}$ per $1.73 \mathrm{~m}^{2}$. Patients with serum creatinine level of $\geq 6 \mathrm{mg} / \mathrm{dL}$, eGFR of $\leq 15 \mathrm{~mL} / \mathrm{min}$; a malignant tumor, liver disease, thyroid dysfunction, sepsis, massive proteinuria (urine protein,
$>3.5 \mathrm{~g} /$ day), inflammatory disorder or being treated with steroids or immunosuppressive agents were excluded from the study.

A venous blood sample $(5 \mathrm{~mL})$ was drawn with full aseptic precaution after an overnight fast. The blood was centrifuged at 5,000 rpm for 10 minutes at room temperature, serum was separated for routine hematology. Biochemistry, urinalysis, and urine protein measurements were performed as per study protocols. An automated blood-cell analyzer (BC-5380; Mindray, Shenzhen, People's Republic of China) was used for routine hematology testing, and an automated clinical biochemistry analyzer (Cobas C 311; Roche-Hitachi, Tokyo, Japan) was used for blood urea nitrogen, creatinine, uric acid, serum lipids, electrolytes, and albumin. Kidney biopsy was performed in those subjects who had provided written consent. Enzyme-linked immunosorbent assay (ELISA) technique was used to measure urine NGAL (uNGAL) levels. A clean, morning midstream urine sample $(5 \mathrm{~mL})$ was collected into a sterile test tube and centrifuged at 5,000 rpm for 15 minutes. The supernatant was transferred to an Eppendorf tube and stored at $-80^{\circ} \mathrm{C}$ until assessment for uNGAL. A human NGAL ELISA kit (Epitope Diagnostics, Inc., San Diego, CA, USA) was used for estimation of uNGAL as per manufacturer's protocol.

\section{Follow-up and end-points}

Baseline renal function tests for all patients were recorded at the time of enrollment. All of the patients had follow-up visits at the outpatient clinic or by interviews telephonically at monthly intervals, and serial renal function tests were repeated at 3-month intervals. The primary follow-up end-point was 18 months, or eGFR of less than $15 \mathrm{~mL} / \mathrm{min}$. Secondary follow-up end-point was number of subjects who expired during this period.

\section{Statistical analysis}

Continuous variables were expressed as mean \pm standard deviation and compared using one way analysis of variance followed by Tukey's post hoc tests. Correlations between various kidney function parameters had been calculated using Pearson correlation coefficient. Receiver operator curve analysis was done for log NGAL and identifying the optimal NGAL cut-off values for predicting progression of CKD. The effects of the cut-off points of log NGAL values, on the survival of the study subjects were assessed using Kaplan-Meier method. Statistical significance was set at $P<0.05$. All the analyses were done using SPSS 20.0 and MedCalc software. 
Table I Demographic characteristics of the study subjects

\begin{tabular}{|c|c|c|c|c|}
\hline Parameters & $\begin{array}{l}\text { Stage II } \\
(n=27)\end{array}$ & $\begin{array}{l}\text { Stage III } \\
(n=33)\end{array}$ & $\begin{array}{l}\text { Stage IV } \\
(n=30)\end{array}$ & $P$-value \\
\hline \multicolumn{5}{|l|}{ Sex } \\
\hline Male (n\%) & $13(48.1)$ & $16(48.5)$ & $16(53.4)$ & $P=0.90$ \\
\hline Female (n\%) & 14 (5I.9) & $17(5 \mid .5)$ & $14(46.6)$ & $P=0.91$ \\
\hline Age (years) & $43.37 \pm 11.46$ & $45.42 \pm 12.30$ & $44.35 \pm 11.34$ & $P=0.80$ \\
\hline BMI $\left(\mathrm{kg} / \mathrm{m}^{2}\right)$ & $20.75 \pm 1.81$ & $20.26 \pm 2.50$ & $19.44 \pm 1.83$ & $P=0.06$ \\
\hline $\mathrm{DBP}(\mathrm{mmHg})$ & $73.93 \pm 8.67$ & $90.31 \pm 8.01$ & $87.09 \pm 4.64$ & $P<0.00$ I \\
\hline $\mathrm{SBP}(\mathrm{mmHg})$ & $\mid 27.48 \pm 7.43$ & $|44.8| \pm 5.63$ & $151.88 \pm 8.0$ & $P<0.001$ \\
\hline
\end{tabular}

Abbreviations: BMI, body mass index; DBP, diastolic blood pressure; SBP, systolic blood pressure.

\section{Results}

This study was performed on $90 \mathrm{CKD}$ subjects of different stages ( stage II $=27$, stage III $=33$, stage IV $=30$ ) and mean age was $43.37 \pm 11.46$ years for stage II, $45.42 \pm 12.30$ years for stage III, and $44.35 \pm 11.34$ years for stage IV respectively (Table 1). Blood urea, serum creatinine, and serum uric acid levels, were significantly increased with disease severity (Table 2). Kidney biopsy was performed in 76 patients $(84.5 \%)$, while 14 patients $(15.5 \%)$ refused to undergo this procedure. Membranous glomerulonephritis: 26 (34.2\%), minimal change disease: 20 (26.3\%), membranoproliferative: 15 (19.7\%), and focal segmental disease: $15(19.8 \%)$ were the leading causes of glomerulonephritis. In the present study, correlation of uNGAL with various parameters including serum creatinine, serum uric acid, and $\mathrm{Ca} \times \mathrm{PO} 4$ showed a significant positive correlation $(r=>0.30)$, whereas eGFR, total cholesterol, and serum phosphorus showed significant inverse correlation (Figure 1).
Baseline uNGAL levels were $\log 1.78 \pm 2.08 \mathrm{ng} / \mathrm{mL}$ in stage II, $\log 3.34 \pm 2.74 \mathrm{ng} / \mathrm{mL}$ in stage III, and $\log 3.70 \pm 0.18$ $\mathrm{ng} / \mathrm{mL}$ in stage IV. Progressor subjects presented with significantly increased uNGAL values at baseline compared with non-progressors. Mean value of UNGAL in progressors (stage II $=3.37 \pm 0.20 \mathrm{ng} / \mathrm{mL}$, stage III $=4.98 \pm 0.19 \mathrm{ng} / \mathrm{mL}$, stage IV $=6.77 \pm 0.16 \mathrm{ng} / \mathrm{mL}$ ) were higher, whereas mean value of UNGAL in non-progressors was (stage II $=3.09 \pm 1$. 10 $\mathrm{ng} / \mathrm{mL}$, stage III $=4.04 \pm 1.80 \mathrm{ng} / \mathrm{mL}$, stage IV $=4.406 \pm 2.13$ $\mathrm{ng} / \mathrm{mL}$ ) lower (Table 3 ). This showed that progressors had a high value of UNGAL at base line. Receiver operator curve analysis for NGAL considering the progression of CKD as status variable was carried out. The area under the curve for uNGAL was 0.878 (95\% confidence interval: $0.68-0.96)$. At the cut-off value of uNGAL $\log 3.5$ unit there was sensitivity of $93.08 \%$ and specificity of $71.43 \%$ in predicting the progression of CKD. Above this value patients experienced a significantly faster disease progression as observed during follow-up time of 18 months (Figure 2).

Regression analysis was performed using eGFR as the dependent variable in a multiple regression model including covariate, as reported in univariate analysis. The associations with $\log$ uNGAL $(\beta=0.38, P<0.001), \mathrm{Ca} \times \mathrm{PO} 4(\beta=0.60$, $P<0.001)$, hemoglobin $(\beta=0.37, P<0.001)$, urine protein ( $\beta=0.34, P<0.001)$, serum albumin $(\beta=0.48, P<0.001)$, and systolic blood pressure $(\beta=0.76, P<0.001)$ were found to be significant in the analysis. The model explains $70.3 \%$ of the total variation in eGFR (Table 4).

A total of ten (22.7\%) patients died during the follow-up period, out of these three patients died due to myocardial injury. The other seven patients experienced cardiovascular

Table 2 Biochemical characteristics of the study subjects

\begin{tabular}{|c|c|c|c|c|}
\hline Parameters & Stage II $(n=27)$ & Stage III $(n=33)$ & Stage IV $(n=30)$ & $P$-value \\
\hline eGFR $\left(\mathrm{mL} / \mathrm{min} / \mathrm{l} .73 \mathrm{~m}^{2}\right)$ & $69.01 \pm 7.52$ & $40.94 \pm 8.51$ & $23.67 \pm 5.30$ & $P<0.00$ I \\
\hline Blood urea (mg\%) & $40.11 \pm 4.58$ & $46.52 \pm 12.22$ & $79.16 \pm 43.74$ & $P<0.001$ \\
\hline Serum creatinine (mg\%) & $1.18 \pm 0.19$ & $1.63 \pm 0.56$ & $2.89 \pm 0.80$ & $P<0.00$ I \\
\hline Serum albumin (mg\%) & $4.0 \mathrm{I} \pm 0.36$ & $3.85 \pm 0.36$ & $3.56 \pm 0.44$ & $P<0.00$ I \\
\hline $\mathrm{Hb}(\mathrm{gm} \%)$ & $9.57 \pm 1.4 \mid$ & $9.16 \pm 1.66$ & $8.09 \pm 1.26$ & $P<0.01$ \\
\hline Serum uric acid (mg\%) & $3.55 \pm 0.48$ & $4.89 \pm 1.44$ & $8.45 \pm 2.94$ & $P<0.00$ I \\
\hline Serum protein (mg\%) & $5.75 \pm 0.73$ & $5.9| \pm 0.7|$ & $5.12 \pm 0.90$ & $P<0.00$ I \\
\hline $\mathrm{Ca}++(\mathrm{mg} \%)$ & $8.25 \pm 1.35$ & $7.17 \pm 1.07$ & $7.77 \pm 1.27$ & $P<0.01$ \\
\hline PO4- (mg\%) & $3.01 \pm 0.55$ & $3.82 \pm 1.02$ & $7.8 \mathrm{I} \pm 2.20$ & $P<0.00 I$ \\
\hline $\mathrm{Ca} \times \mathrm{PO} 4$ & $25.07 \pm 6.60$ & $27.16 \pm 8.06$ & $61.42 \pm 22.91$ & $P<0.001$ \\
\hline LDL (mg\%) & $108.85 \pm 28.62$ & $90.21 \pm 17.86$ & $89.14 \pm 35.82$ & $P<0.05$ \\
\hline HDL (mg\%) & $38.4 I \pm 9.45$ & $39.48 \pm 6.24$ & $4 I .4 I \pm 8.67$ & $P=0.37$ \\
\hline TG (mg\%) & $98.78 \pm 41.41$ & $99.52 \pm 19.86$ & $121.94 \pm 20.96$ & $P<0.0$ I \\
\hline $\mathrm{TC}(\mathrm{mg} \%)$ & $223.63 \pm 49.48$ & $|55.2| \pm 28.54$ & $167.79 \pm 55.68$ & $P<0.001$ \\
\hline 24 hours urine protein (mg\%) & $361.44 \pm 257.37$ & $570.27 \pm 616.28$ & $659.44 \pm 750.96$ & $P=0.16$ \\
\hline
\end{tabular}

Abbreviations: eGFR, estimated glomerular filtration rate; Hb, hemoglobin; LDL, low-density lipoprotein; HDL, high-density lipoprotein; TG, triglycerides; TC, total cholesterol. 


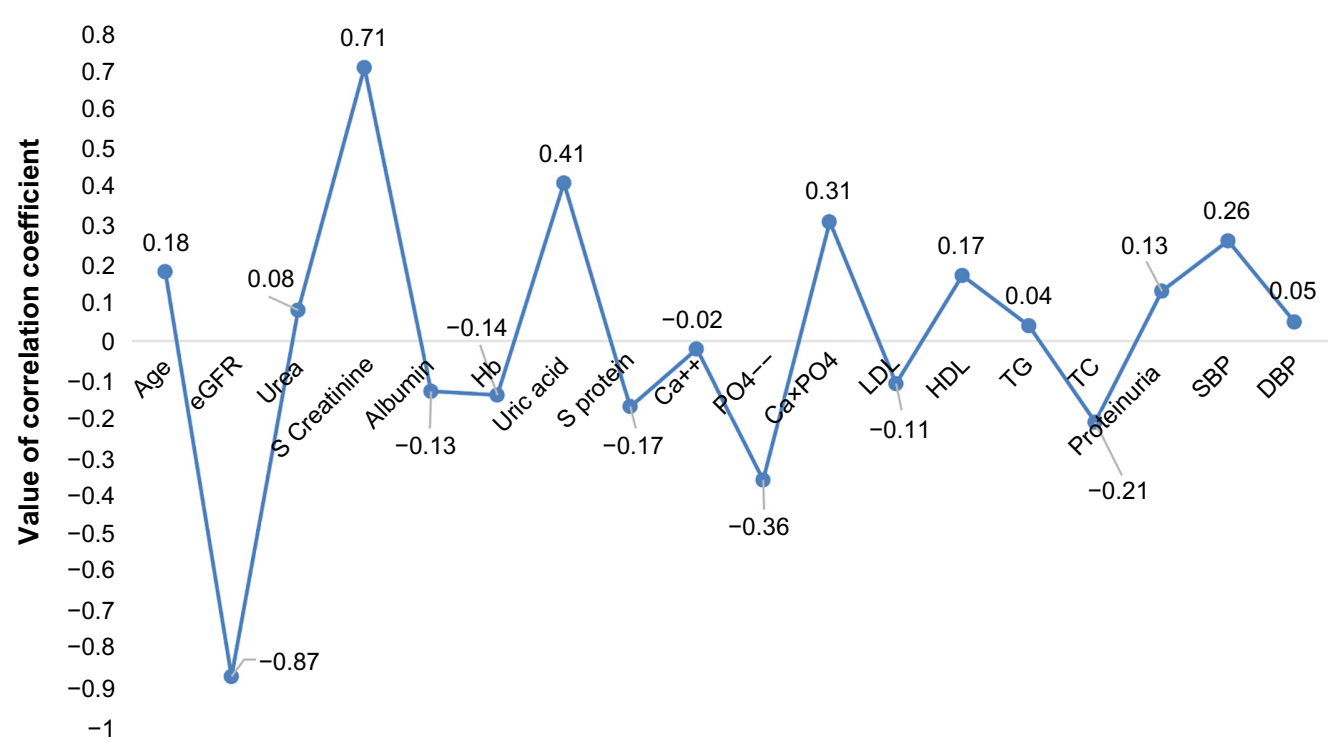

Figure I Correlation coefficient of log NGAL with various demographic and biochemical parameters.

Abbreviations: eGFR, estimated glomerular filtration rate; Hb, hemoglobin; LDL, low-density lipoprotein; HDL, high-density lipoprotein; TG, triglycerides; SBP, systolic blood pressure; DBP, diastolic blood pressure; TC, total cholesterol.

events such as sudden cardiac deaths, atrial-ventricular block, and congestive heart failure during the follow-up period of 18 months. Kaplan-Meier survival analysis showed NGAL to be closely associated with the renal end-point in CKD patients. Based on the log NGAL cut-off of 3.51 unit, patients with uNGAL levels less than 3.51 unit had a renal survival rate of $71.4 \%$ while patients with uNGAL level greater than 3.51 unit had a renal survival rate of $14.7 \%$ (Figure 3 ).

\section{Discussion}

Early detection and treatment of kidney disease can result in preventing kidney disease progression. Thus, an early biomarker of kidney damage which can identify patients at an increased risk of disease progression would be helpful in kidney disease diagnosis and treatment. ${ }^{10}$ Since creatinine is not a sensitive marker of kidney function and eGFR also has some limitations, there is a growing need to find an early marker of kidney damage, newer studies suggest that NGAL has the potential to be an ideal biomarker to detect early kidney damage in patients at risk. ${ }^{10}$ In a recent study, the CKD Consortium Group has shown that GFR estimation by the cystatin $\mathrm{C}$ based eGFR formula best correlated with clinical outcomes. ${ }^{11}$ In another study performed to calculate the equation of eGFR, creatinine-cystatin $\mathrm{C}$ equation and creatinine or cystatin $\mathrm{C}$ alone were compared. ${ }^{12}$ They found that creatinine-cystatin $\mathrm{C}$ equation is better than the other equations based on either of these markers alone and is used to estimate eGFR. Many of the Asian countries have now validated eGFR equations specifically for their respective populations. Although efforts to develop an equation for the Indian population are continuing, we do not have such an equation yet. ${ }^{13} \mathrm{NGAL}$ is synthesized systemically in response to kidney damage. It could also be produced locally by injured tubules. A third source of NGAL may be activated neutrophils/macrophages or inflamed vasculature, frequently found in CKD. ${ }^{14}$

In a recent study elevated fibroblast growth factor-23 was an independent risk factor for end-stage renal disease in patients with relatively preserved kidney function and for mortality across the spectrum of CKD. ${ }^{15}$ However,

Table 3 Disease progression in CKD stage II, III, IV

\begin{tabular}{|c|c|c|c|c|c|c|c|}
\hline Stage of CKD & Number & $\begin{array}{l}\text { Mean log uNGAL } \\
\text { baseline (ng/mL) }\end{array}$ & III & IV & $\mathbf{v}$ & $\begin{array}{l}\text { Mean log uNGAL } \\
\text { (ng/mL) }\end{array}$ & P-value \\
\hline II & 27 & $1.78 \pm 2.08$ & 13 & - & - & $3.37 \pm 0.20$ & $P<0.05$ \\
\hline III & 33 & $3.34 \pm 2.74$ & $x$ & 8 & 7 & $4.98 \pm 0.19$ & $P<0.05$ \\
\hline IV & 30 & $3.70 \pm 0.18$ & $x$ & $X$ & 17 & $6.77 \pm 0.16$ & $P<0.001$ \\
\hline $\mathrm{N}$ & 90 & - & - & - & 24 & - & - \\
\hline
\end{tabular}

Note: Significant increase in mean values of log uNGAL observed for progressors as compared to non-progressors.

Abbreviations: CKD, chronic kidney disease; uNGAL, urinary NGAL; N, total number. 


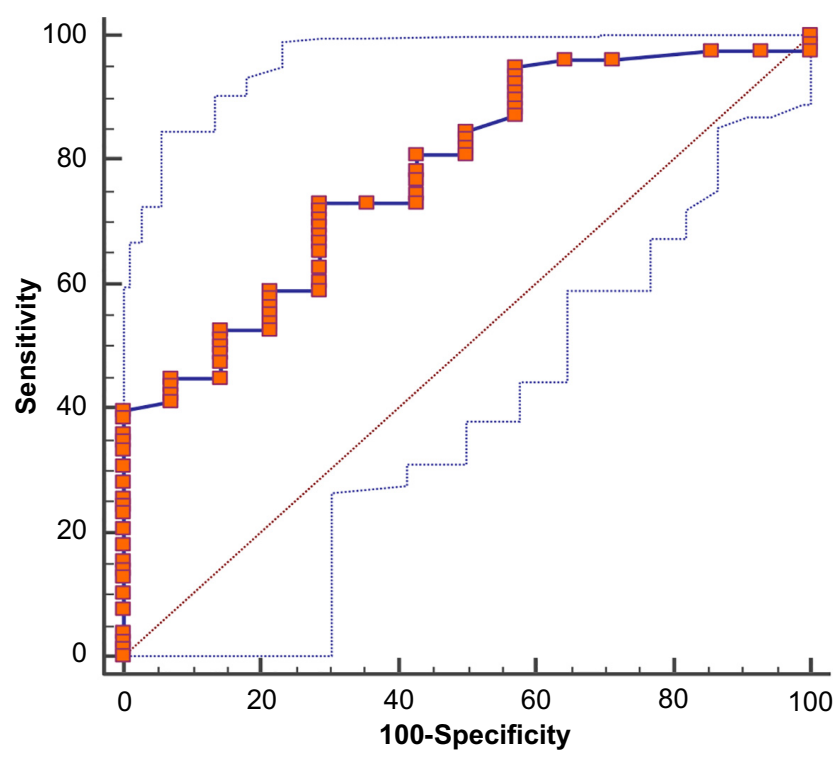

Figure 2 Receiver operator curve for NGAL considering the progression of chronic kidney disease (CKD) as status variable.

Notes: The area under the curve for NGAL was 0.878 ( $95 \%$ confidence interval: 0.68-0.96). A log NGAL cut-off of 3.5 I unit had a sensitivity of $93.08 \%$ and specificity of $71.43 \%$ in predicting the progression of CKD. Orange points are the individual data.

a conflicting result was obtained by the same author in another study which showed that higher fibroblast growth factor-23 levels are not independently associated with higher risk of incident $\mathrm{CKD}$ in patients with type 2 diabetes. ${ }^{16}$ We found that mean uNGAL level was significantly increased with disease severity, this result is consistent with the observation by Bolignano et al who reported higher mean serum NGAL and uNGAL in patients with CKD compared with the healthy control group. ${ }^{17} \mathrm{NGAL}$ would thus represent the expression of how much active kidney damage lies beneath the overall condition of chronic renal impairment, rather than being a simple marker of decreased filtration such as serum creatinine, as recently proposed by Mori and Nakao. ${ }^{18}$ Mitsnefes et al also showed that NGAL could represent a marker of renal function in children with CKD. ${ }^{19}$

Table 4 Regression analysis (univariate and multivariate) of eGFR at baseline

\begin{tabular}{lll}
\hline Parameter & $\boldsymbol{\beta}$ & $\boldsymbol{P}$-value \\
\hline Log UNGAL & 0.38 & $P<0.001$ \\
CaxPO4 & 0.60 & $P<0.001$ \\
Hemoglobin & 0.37 & $P<0.001$ \\
Urine protein & 0.34 & $P<0.001$ \\
Serum albumin & 0.48 & $P<0.00$ I \\
SBP & 0.76 & $P<0.001$ \\
\hline
\end{tabular}

Notes: Multiple $R=0.84, R^{2}=70.3 \% ; \beta$ is the standardized coefficient.

Abbreviations: eGFR, estimated glomerular filtration rate; uNGAL, urinary NGAL; SBP, systolic blood pressure.

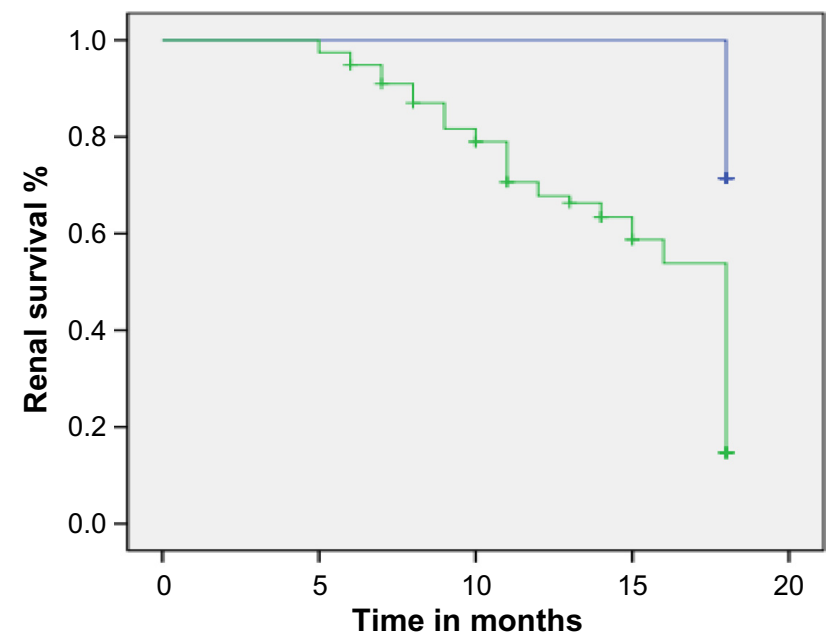

Figure 3 Kaplan-Meier survival curve shows NGAL to be closely associated with the renal end-point in chronic kidney disease patients.

Notes: Based on the log NGAL cut-off of 3.5 I unit, patients with NGAL levels less than 3.5 I unit had a renal survival rate of $71.4 \%$ while patients with NGAL level greater than $3.5 \mathrm{I}$ unit had a renal survival rate of $14.7 \%$. Blue line, patients survival with log uNGAL $<3.5$ I unit. Green line, patients survival with uNGAL >3.5I unit.

In our study NGAL showed significant correlation with eGFR $(r=-0.87)$, proteinuria $(r=0.13, P<0.001)$, and creatinine $(r=0.71, P<0.001)$. Similar results were observed in another study of subjects with CKD (due to chronic glomerulonephritis) which showed that mean uNGAL concentrations were higher in CKD patients. Furthermore, uNGAL concentrations were significantly correlated with eGFR $(r=-0.528$, $P=0.04)$ and proteinuria $(r=0.294, P=0.01) .{ }^{16,20,21}$

In our study no association was found with sex but few studies reported that male sex showed significantly faster progression to end-stage renal disease, ${ }^{22}$ although this association was debated by other authors because it seemed to be strongly confounded by other factors. ${ }^{23}$ Our study showed significant association with calcium-phosphate product $(r=0.31, P<0.01)$ and uric acid $(r=0.41, P<0.001)$. Similar results were observed by other authors that high calcium-phosphate product was associated with an increased independent risk of disease progression in CKD subjects. ${ }^{24}$ Another study was done on 80 non-diabetic patients with CKD stages II to IV, 80 kidney transplant recipients, and 32 healthy control subjects. Study results showed that serum NGAL values were significantly higher in kidney allograft recipients and in CKD patients compared with healthy controls. NGAL rose gradually, reaching the highest value in advanced $\mathrm{CKD}^{25}$

We determined best cut-off values to predict early stage CKD in our study. For uNGAL this value was found to be $3.51 \mathrm{log} \mathrm{ng} / \mathrm{mL}$, with a sensitivity of $93.08 \%$ and specificity of $71.43 \%$ in predicting the progression of CKD. In a study 
by Bolignano et al the best cut-off level for sNGAL was found to be $435 \mathrm{ng} / \mathrm{mL}$ (sensitivity $83.9 \%$, specificity $53.8 \%$ ). ${ }^{17}$

In our study, the best overall agreement with uNGAL was found for eGFR. Moreover, our data suggest that NGAL assay is also able to detect patients with only subclinical ${ }^{26}$ or modest renal damage, which may not be revealed by significant variations in renal function test, such as serum creatinine.

\section{Limitations of our study}

Small sample size, diagnosis of myocardial infarction/angina was based on the clinical evaluation by treating physician and the cause of death was not determined by postmortem examination. It is possible that some of the sudden cardiac deaths were due to hyperkalemia rather than an acute coronary syndrome.

\section{Conclusion}

Our results indicate that uNGAL has a better prognostic value to indicate kidney impairment in CKD subjects.

\section{Disclosure}

The authors have no conflicts of interest to disclose.

\section{References}

1. Hunsicker LG, Adler S, Caggiula A, et al. Predictors of the progression of renal disease in the modification of diet in renal disease study. Kidney Int. 1997;51(6):1908-1919.

2. Haroun MK, Jaar BG, Hoffman SC, et al. Risk factors for chronic kidney disease: a prospective study of 23,534 men and women in Washington County, Maryland. J Am Soc Nephrol. 2003;14(11):2934-2941.

3. Ejerblad E, Fored CM, Lindblad P, et al. Association between smoking and chronic renal failure in a nationwide populationbased case-control study. J Am Soc Nephrol. 2004;15(8):2178-2185.

4. Kurella M, Lo JC, Chertow GM. Metabolic syndrome and the risk for chronic kidney disease among nondiabetic adults. J Am Soc Nephrol. 2005;16(7):2134-2140.

5. Ruggenenti P, Perna A, Mosconi L, et al. Urinary protein excretion rate is the best independent predictor of ESRF in nondiabetic proteinuric chronic nephropathies. 'Gruppo Italiano di Studi Epidemiologici in Nefrologia' (GISEN). Kidney Int. 1998;53(5):1209-1216.

6. Levey AS, Eckardt KU, Tsukamoto Y, et al. Definition and classification of chronic kidney disease: A position statement from Kidney Disease: Improving Global Outcomes (KDIGO). Kidney Int. 2005;67(6):2089-2100.

7. Perrone RD, Madias NE, Levey AS. Serum creatinine as an index of renal function: new insights into old concepts. Clin Chem. 1992; 38(10):1933-1953.

8. Shemesh O, Golbetz H, Kriss JP, Myers BD. Limitations of creatinine as a filtration marker in glomerulopathic patients. Kidney Int. 1985; 28(5):830-838.
9. Garabed Eknoyan. KDIGO Clinical Practice Guideline for Anemia in Chronic Kidney Disease. 2012;2(4):279-335.

10. Barasch JM, Devarajan P, Nickolas TL, Mori K. Detection of NGAL in chronic renal disease. Google Patents US 8592170 B2; 2005.

11. Shlipak MG, Matsushita K, Ärnlöv J, et al. Cystatin C versus creatinine in determining risk based on kidney function. $N$ Engl $J$ Med. 2013;369(10):932-943.

12. Inker LA, Schmid CH, Tighiouart $\mathrm{H}$, et al. Estimating glomerular filtration rate from serum creatinine and cystatin C. N Engl J Med. 2012; 367(1):20-29.

13. Bhowmik D, Agrawal A, Panda S. Assessing the prevalence of chronic kidney disease in the community: Estimating glomerular filtration rate is the Achilles heel. Indian J Nephrol. 2014;24(6):411-412.

14. Schmidt-Ott KM, Mori K, Kalandadze A, et al. Neutrophil gelatinaseassociated lipocalin-mediated iron traffic in kidney epithelia. Curr Opin Nephrol Hypertens. 2006;15(4):442-449.

15. Isakova T, Xie H, Yang W, et al. For the Chronic Renal Insufficiency Cohort (CRIC) Study Group; Fibroblast Growth Factor 23 and Risks of Mortality and End-Stage Renal Disease in Patients With Chronic Kidney Disease. JAMA. 2011;305(23):2432-2439.

16. Isakova T, Craven TE, Lee J, et al. Fibroblast growth factor 23 and incident CKD in type 2 diabetes. Clin J Am Soc Nephrol. 2015;10(1):29-38.

17. Bolignano D, Lacquaniti A, Coppolino G, et al. Neutrophil gelatinaseassociated lipocalin (NGAL) and progression of chronic kidney disease. Clin J Am Soc Nephrol. 2009;4(2):337-344.

18. Mori K, Nakao K. Neutrophil gelatinase-associated lipocalin as the real-time indicator of active kidney damage. Kidney Int. 2007;71(10): 967-970.

19. Mitsnefes MM, Kathman TS, Mishra J, et al. Serum neutrophil gelatinase-associated lipocalin as a marker of renal function in children with chronic kidney disease. Pediatr Nephrol. 2007;22(1):101-108.

20. Bolignano D, Coppolino G, Campo S, et al. Urinary neutrophil gelatinase-associated lipocalin (NGAL) is associated with severity of renal disease in proteinuric patients. Nephrol Dial Transplant. 2008;23(1):414-416.

21. Bolignano D, Lacquaniti A, Coppolino G, Campo S, Arena A, Buemi M. Neutrophil gelatinase-associated lipocalin reflects the severity of renal impairment in subjects affected by chronic kidney disease. Kidney Blood Press Res. 2008;31(4):255-258.

22. Neugarten J, Acharya A, Silbiger SR. Effect of gender on the progression of nondiabetic renal disease: A meta-analysis. J Am Soc Nephrol. 2000;11(2):319-329.

23. Jafar TH, Schmid CH, Stark PC, et al. The rate of progression of renal disease may not be slower in women compared with men: A patient-level meta-analysis. Nephrol Dial Transplant. 2003;18(10):2047-2053.

24. Schwarz S, Trivedi BK, Kalantar-Zadeh K, Kovesdy CP. Association of disorders in mineral metabolism with progression of chronic kidney disease. Clin J Am Soc Nephrol. 2006;1(4):825-831.

25. Malyszko J, Malyszko JS, Bachorzewska-Gajewska H, Poniatowski B, Dobrzycki S, Mysliwiec M. Neutrophil gelatinase-associated lipocalin is a new and sensitive marker of kidney function in chronic kidney disease patients and renal allograft recipients. Transplant Proc. 2009;41(1):158-161.

26. Sachan R, Patel ML, Gaurav A, Angwar R. Serum Neutrophil Gelatinase-Associated Lipocalin: Predictor of Pre-Eclampsia, Eclampsia and Its Correlation with Severity of Disease. International Journal Medicine and Medical Sciences. 2013;46(2):1170-1178.

\section{Publish your work in this journal}

The International Journal of Nephrology and Renovascular Disease is an international, peer-reviewed open-access journal focusing on the pathophysiology of the kidney and vascular supply. Epidemiology, screening, diagnosis, and treatment interventions are covered as well as basic science, biochemical and immunological studies. The journal welcomes original research, clinical studies, reviews \& evaluations, expert opinion and commentary, case reports and extended reports. The manuscript management system is completely online and includes a very quick and fair peerreview system, which is all easy to use. Visit http://www.dovepress.com/ testimonials.php to read real quotes from published authors. 